
\title{
25 Research Square \\ Regional and Age Epidemiological Analysis of Hepatitis A Viral in China, 2004-2017
}

\section{Yan Xu}

Hunan Aerospace Hospital

Ruiti Tang

Hunan Aerospace Hospital

Weiming Li ( $\nabla$ liweiming@csu.edu.cn )

Huazhong University of Science and Technology Union Shenzhen Hpspital https://orcid.org/00000003-1313-9688

\section{Research Article}

Keywords: HAV, HAV infection, Epidemiology

Posted Date: September 20th, 2021

DOI: https://doi.org/10.21203/rs.3.rs-864630/v1

License: (c) (1) This work is licensed under a Creative Commons Attribution 4.0 International License. Read Full License 


\section{Abstract}

Background: To describe the prevalence features, including age, regions, morbidity and mortality of hepatitis A viral in China from 2004 to 2017 by searching the China Public Health Sciences Data Center with the keyword "Hepatitis A virus (HAV)".

Methods: In this study, HAV morbidity and mortality data were retrieved from China Public Health Science Data Center (CPHSD) using HAV as a keyword. HAV infection data from 2004 to 2017, HAV cases and HAV-related deaths were retrieved from 31 regions in China, except Taiwan, Hong Kong and Macau. SPSS 18 is used for statistical analysis. GraphPad Prism 5 is used to draw line graphs and histograms. Microsoft PowerPoint 2016 and Adobe Illustrator CS 6 are used for drawing geographically distributed China maps.

Results: From 2004 to 2017, the rates of hepatitis A viral morbidity and mortality in China were keeping decrease annually (7.1997 and 3.0772 in 2004; 2.6430 and 0.2997 in 2010; 1.3679 and 0.2899 in 2017, respectively, $1 / 100,000)(p<0.001)$. The HAV infection rate of children $(0-10$ years old) was higher than that of the elderly (>50 years old) $(p<0.001)$. The geographical distribution of HAV prevalence showed significant regional differences which showed that hepatitis A patients were more prevalent in Sichuan and Xinjiang provinces (averaged number $>4,000$ / year). HAV-related death does not differ much between regions (averaged number 0 2.1/ year)

Conclusions: Our analysis of viral hepatitis A prevalence features over past 14 years suggests that the incidence and mortality of HAV are decreased annually in China.

\section{Introduction}

HAV (Hepatitis A virus), one of the world's most common infected virus, is a plus-strand RNA virus, belonging to the Picornaviruses family of Hepatoviruses. HAV was discovered by Feinslone et al. (1973) in the feces of patients at the acute stage by immunoelectron microscopy.[1] HAV is spherical with a diameter of about $27 \mathrm{~nm}$. The capsid of HAV has a 20-hedral symmetrical structure and is composed of 60 shell particles. Shell particles are composed of four kinds of peptides, VP1, VP2, VP3 and VP4.[2] HAV can cause symptomatic or asymptomatic infections in humans.

HAV infection is acquired primarily through the faecal-oral route, including close person-to-person contact and ingestion of food or water contaminated by the faeces of an infected person.[3] In rare cases, HAV infection may also be transmitted by receiving blood products from an infected blood donor.[4, 5] According to the prevalence of HAV, it can be divided into high, medium, low and very low HAV endemic areas. In highly epidemiological areas, most people are infected with HAV in early childhood. The first exposure of adults in low-prevalence areas to HAV is usually due to travel and living in areas with high prevalence of HAV, or engaging in risky behaviours, such as contact with infected persons, male-to-male sex (MSM), or the use of illicit drugs.[5-7] 
With the widespread vaccination of HAV vaccine worldwide and the improvement of sanitary conditions, the epidemic areas of HAV show a trend of epidemiological decline, and the prevalence of HAV has been effectively controlled.[8, 9] In the past 20 years, HAV vaccination and lifestyle changes have both contributed to a rapid decline in HAV risk in China. Lifestyle changes are likely to have the biggest effect on reducing HAV risk in regions with the fastest GDP growth.[9] Reports from 2004 to 2009 show that most HAV cases in China are concentrated in Henan, Sichuan, Yunnan, Guizhou, Xinjiang and Gansu provinces. The number of HAV cases in those six provinces accounted for $44 \% \sim 51 \%$ of the total cases in China during the investigation time. Nearly $30 \%$ of cases are children younger than 15 years of age.[10] HAV public health emergencies are mainly concentrated in primary and secondary schools, and the cases are mainly in school students.[11] The influence of regional and age differences on the clinical epidemiology of HAV was significant.[7]

The aim of this study is to investigate the incidence and mortality of HAV in China from 2004 to 2017, and to analyze the regional and age characteristics of patients with hepatitis $A$, so as to provide additional guidance for the government and improve the awareness of prevention and control of HAV in China.

\section{Methods}

HAV cases is an infectious disease of liver inflammation caused by HAV infection. Clinical fatigue, loss of appetite, hepatomegaly and abnormal liver function are the main manifestations. Some cases showed jaundice, mainly acute hepatitis. In addition, asymptomatic infection is more common. HAV related death is defined as all deaths from clinical diseases caused by viral hepatitis A, such as liver failure, hepatic encephalopathy, hepatorenal syndrome, and infection.

In this study, HAV morbidity and mortality data were retrieved from China Public Health Science Data Center (CPHSD) using HAV as a keyword[12]. HAV infection data from 2004 to 2017, HAV cases and HAVrelated deaths were retrieved from 31 regions in China, except Taiwan, Hong Kong and Macau. The morbidity and mortality of HAV reported by CPHSD is equal to infectious diseases/population $\times 100,000$; Deaths/population $\times 100,000$. Grouping analysis by age and region was conducted to investigate differences in the prevalence of HAV.

The corresponding data expressed as "mean (standard deviation, SD)", "mean (standard error, SE)" or percentage learn as appropriate. For normally distributed continuous variables, student's t-test and oneway ANCOVA were performed to analyze the differences. The $p$ value $<0.05$ is considered a statistically significant difference. SPSS 18 is used for statistical analysis. GraphPad Prism 5 is used to draw line graphs and histograms. Microsoft PowerPoint 2016 and Adobe Illustrator CS 6 are used for drawing geographically distributed China maps.

\section{Results}




\section{HAV in China}

This study retrospectively analyzed HAV data published by the Chinese government (2004-2017). It was found that both the incidence of HAV $(1 / 100,000)$ and HAV-related death $(1 / 100,000)$ showed a decreasing trend year by year (Fig. 1)., which show that the incidence of HAV and HAV-related death were 7.1997 and 3.0772 in 2004, 2.6430 and 0.2997 in 2010, and 1.3679 and 0.2899 in 2017 (Fig. 1, Table 1). The results of linear regression analysis show good consistency $\left(r^{2}>0.719, p<0.05\right)$. In addition, from 2004 to 2017, there were 614,857 cases of HAV "43,918(24,184)" and 231 deaths due to HAV-related death "16.5(14.2)".

Figure 1. (A) The incidence of HAV cases (1/100,000) in Chinese populations, 2004-2017.(B) The incidence of HAV-related death $(1 / 100,000)$ in Chinese populations, 2004-2017.

Table 1

The data of HAV cases, HAV-related death in China, 2004 to 2017.

\begin{tabular}{|lllll|}
\hline Year & \multicolumn{2}{l}{ HAV cases } & \multicolumn{2}{l|}{ HAV-related death } \\
\hline & Number & Morbidity $(\mathbf{1 / 1 0 0 , 0 0 0 )}$ & Number & Morbidity $(\mathbf{1 / 1 0 0 , 0 0 0 )}$ \\
\hline 2004 & 93587 & 7.1997 & 40 & 3.0772 \\
\hline 2005 & 73349 & 5.6428 & 43 & 3.3080 \\
\hline 2006 & 68667 & 5.2515 & 37 & 2.8297 \\
\hline 2007 & 77135 & 5.8681 & 29 & 2.2062 \\
\hline 2008 & 56052 & 4.2422 & 10 & 0.7568 \\
\hline 2009 & 43841 & 3.3012 & 21 & 1.5813 \\
\hline 2010 & 35277 & 2.6430 & 4 & 0.2997 \\
\hline 2011 & 31456 & 2.3459 & 13 & 0.9695 \\
\hline 2012 & 24453 & 1.8149 & 5 & 0.3711 \\
\hline 2013 & 22244 & 1.6428 & 2 & 0.1477 \\
\hline 2014 & 25969 & 1.9163 & 8 & 0.5903 \\
\hline 2015 & 22667 & 1.6637 & 10 & 0.7340 \\
\hline 2016 & 21285 & 1.5528 & 5 & 0.3648 \\
\hline 2017 & 18875 & 1.3679 & 4 & 0.2899 \\
\hline & & & & \\
\hline
\end{tabular}

\section{Regional Difference Of Hav Prevalence}


The study included 31 regions in China. We mapped histogram and geographical distribution to show regional differences in HAV incidence / HAV-related death (Fig. 2). According to the data on the number of HAV cases, the top three provinces with the highest number of HAV cases are Sichuan, Xinjiang and Yunnan, of which Sichuan and Xinjiang have an average number (year) of more than 4,000 (Fig. 2A, 2B). The average number (year) by regional distribution of HAV-related death in 31 regions is between 0-2.1. It does not differ much between regions. Among them, Guizhou means is 2.07 , which is relatively the highest (Fig. 2C, 2D). In Beijing, the incidence of HAV decreased from 0.2093 in January 2004 to 0.0552 in January $2017(p<0.05)$. The reduction rate was also observed in regions with the highest HAV cases, such as Xinjiang and Sichuan provinces $(p<0.05)$.

(A) (B) The average number (year) by regional distribution of HAV cases; (C) (D) The average number (year) by regional distribution of HAV-related death. The coordinate numbers on the $\mathrm{X}$ axis of the Histogram (A) (C) represent different regions: 1-Beijing, 2-Tianjin, 3-Hebei, 4-Shanxi, 5-Inner Mongolia, 6Liaoning, 7-Jilin, 8-Heilongjiang, 9-Shanghai, 10-Jiangsu, 11-Zhejiang, 12-Anhui, 13-Fujian, 14-Jiangxi, 15Shandong, 16-Henan, 17-Hubei, 18-Hunan, 19-Guangdong, 20-Guangxi, 21-Hainan, 22-Chongqing, 23Sichuan, 24-Guizhou, 25-Yunnan, 26-Tibet, 27-Shaanxi, 28-Gansu, 29-Qinghai, 30-Ningxia, 31-Xinjiang.

\section{Age Differences Of Hav Prevalence}

To investigate the age difference in HAV prevalence, we first divided the cases into three categories: 0-20 years old, 20-50 years old and $>50$ years old. It was found that the number of HAV cases have the highest trend in 20-50 years old group, which was significantly higher than the $>50$ years old group ( $p<$ 0.05). The number of HAV-related death cases have the highest trend in the $>50$ years old group, and it was significantly higher than that of $0-20$ years old group $(p<0.05)$ (Fig. 3).

To further investigate the relationship between age and morbidity and mortality, we divided cases aged 0-80 years into several age ranges on a 10-year scale. The results showed that the incidence of HAV decreased with the increase of age. Among them, the HAV cases of patients aged 0-10 years old group was the highest, which was significantly higher than that in 50-60 years old group, 60-70 years old group,70-80 years old group, and $>80$ years old group $(p<0.05)$. The number of HAV-related death cases was highest in the 40-50 years old group and lowest in the 20-30 years old group. The difference in number of HAV-related death cases was significantly between the 40-50 years old group and the 20-30 years old group. In addition, although the incidence of HAV case was significantly higher in the 70-80 years old group than in $>80$ years old group $(p<0.05)$, but there was no significant difference in number of HAV-related death between the two group $(p<0.5)$ (Fig. 4).

(A) HAV cases in Chinese populations, 2004-2017; (B) HAV-related death in Chinese populations, 20042017; (C) The percent distribution of HAV cases and HAV-related death.

(A) HAV cases in Chinese populations, 2004-2017; (B) HAV-related death in Chinese populations, 20042017. 


\section{Discussion}

Based on the analysis of publicly available data from CPHSD, the annual prevalence of HAV and HAVrelated death rates in China have been decreasing. Morbidity and mortality vary by region and by age group. The morbidity rate was the highest among children and adolescents, and the mortality rate was the highest among middle-aged and elderly people (40-50 years old group). The highest morbidity rate was found in Sichuan and Xinjiang provinces. Although the incidence of hepatitis $A$ is decreasing year by year (Fig. 1) (Table 1). However, due to China's vast territory, regional economic development is uneven. And it can be found from Fig. 2 that the areas with high HAV incidence are mainly concentrated in the west, while the population in the west of China is much smaller than that in the southeast. Relatively poor economic conditions and sanitary conditions may be one of the reasons for the high incidence of HAV in the western region (Sichuan, Xinjiang, Yunnan etc.).

The prevalence of HAV, especially in developing countries, remains a great challenge for the world's public health authorities[6].But, in this study, the year-on-year decrease in the prevalence of HAV and HAV-related mortality indicates that China's prevention and control measures have played a positive role. It is known that the prevalence of HAV is closely related to economic conditions and hygiene habits. For more than 10 years, with the development of China's economy, health and medical conditions have gradually improved, residents' awareness of disease prevention has increased, and the promotion of hepatitis A vaccine. These are probably the reasons why the fatality rate and infection rate in China have been declining year by year.

HAV is the most common pathogen that causes acute liver disease worldwide. In 2010, there were 1.4 million cases of hepatitis $A$ and 27,731 deaths worldwide. [13]The incidence of HAV widely between countries, with high prevalence in developing countries. It is also related to the quality of local sanitation, the quality of drinking water sanitation and socio-economic factors. In endemic areas, the severity of the disease is closely related to age. HAV infection mainly occurs in early childhood and, although mostly asymptomatic, can lead to illness and death in some cases. Symptomatic disease often occurs in older infected children and infected adults.[14] For developed areas, due to health, medical care, high economic level, low prevalence. However, herd immunity is low and outbreaks are often reported.[15]

HAV often causes symptomatic hepatitis in adults and asymptomatic subclinical infection in children. The incubation period for HAV is about 28 days. The clinical presentation usually occurs after the incubation period with sudden onset. Signs and symptoms of infection include nausea, vomiting, diarrhea, dark urine, jaundice, fever, headache, weight loss, abdominal pain, and loss of desire to smoke or drink alcohol. The likelihood of symptoms increases with age. Most children under the age of 6 have no symptoms. Jaundice can occur in more than 70 percent of older children and adults infected with HAV. Enlargement of the liver and spleen may occur. Acute illness from HAV infection usually lasts no more than two months. The clinical symptoms of HAV overlap with many other gastrointestinal and febrile symptoms, making it difficult to differentiate from other types of acute viral hepatitis.[16, 17] 
Water-borne HAV infections are mainly due to drinking water contaminated by adjacent septic tanks or swimming in water contaminated with sewage.[18, 19] Food transmission mainly occurs when the faeces of HAV carriers are transferred to food during food preparation, or when the food plants are contaminated by the faeces of HAV carriers during harvesting or processing. Another modality of the faecal-oral route is extensive and close personal contact between the carrier and the vulnerable person. For example, MSM, in closed institutions such as schools and day-care attendance centers are susceptible to HAV by sharing items.[20, 21]

Therefore, the fecal-oral transmission of HAV can be effectively reduced in terms of daily diet, such as heating food and water to 85 degrees Celsius or above for one minute, avoiding contact with and eating uncooked food and natural water.[22, 23] In addition, prevention of vulnerable populations and children through active or passive immunization is the most important approach. Passive immunity: immunoglobulin. It can be used for pre-exposure prophylaxis or, in some cases, post-exposure prophylaxis. And according to the dose of different, in different time has a protective effect.[24] Active immunization: Vaccines. The approval and widespread use of the 1990s HAV vaccine has paved the way for the prevention of this disease. The initial focus was on immunizing children in high prevalence areas and certain other high-risk groups. $[25,26]$ In 2006, the Centers for Disease Control and Prevention (CDC) made a major change, recommending HAV vaccination for all children between 12 and 23 months of age. [27] In China, HAV vaccine has become one of the main vaccines for children and was included in the Expanded Immunization Vaccine for Children in May 2008.

As a developing country with a population of 1.4 billion, China faces great challenges in dealing with the spread of HAV. In 1988, an outbreak of hepatitis A sickened nearly 300,000 people in Shanghai, China, by eating raw clams, a popular delicacy that had been consumed by the city's residents in the month before the outbreak began[28]. With the improvement of economic level and the improvement of residents' environmental hygiene, as well as the widespread vaccination of HAV vaccine throughout the country, the reported incidence of hepatitis A decreased from $>50 / 100,000$ in 1990 to 1.55/100,000 in 2017.[9] Our study found that the incidence of HAV was regional and age specific, and the highest incidence of HAV was found in Xinjiang and Sichuan. The incidence is manifested by age in children, adolescents are vulnerable to infection transmission. The number of death cases showed a relatively high trend in the 40-50 years old group, which was significantly higher than the $20-30$ years old group.

There are some limitations in this study. The coverage of data collection is not high enough to cover the entire HAV-infected population in China, and the lack of access to HAV epidemiological data in Taiwan, Hong Kong and Macau may lead to bias in this study. Also, the public data studies here are incomplete, making it impossible to analyze other risk factors such as race, occupation, residential environment and blood donation. This study focuses on regional and age differences in HAV in mainland China and concludes that HAV has regional and age characteristics. Regional attention needs to be paid to the spread of HAV in Sichuan and Xinjiang. Attention needs to be paid to transmission among children and adolescents. The public should be aware that more effective prevention strategies should be implemented to reduce and prevent the incidence of HAV/hepatitis A disease. 


\section{Conclusions}

This study analyzed the epidemiological characteristics of HAV. The incidence and mortality of HAV are decreasing year by year. The incidence of HAV varies by region and age. In terms of region, the higher incidence of HAV was found in Xinjiang and Sichuan (averaged number $>4,000$ / year). HAV-related death does not differ much between regions (averaged number $0 \sim 2.1$ / year). In terms of age, children and adolescents are vulnerable to transmission. The HAV cases of patients aged 0-10 years old group was the highest. And the number of HAV cases has a downward trend with age. The number of HAV-related death cases showed a relatively high trend in the 40-50 years old group, which was significantly higher than the 20-30 years old group. The results may provide basic data for preventing and controlling the spread of HAV. Regional attention should be paid to the spread of HAV in Xinjiang and Sichuan. Attention needs to be paid to transmission among children and adolescents.

\section{Abbreviations}

CPHSD

China Public Health Science Data Center

$\mathrm{CDC}$

Centers for Disease Control and Prevention

HAV

Hepatitis A virus

MSM

male-to-male sex

\section{Declarations}

Availability of data and materials: In this study, HAV morbidity and mortality data were retrieved from China Public Health Science Data Center (CPHSD) using HAV as a keyword [12]. HAV infection data from 2004 to 2017, HAV cases and HAV-related deaths were retrieved from 31 regions in China, except Taiwan, Hong Kong and Macau.

Authors' contributions: Yan Xu designed the study, implemented the research plan, collected and analyzed the data, and wrote the first manuscript. Ruiti Tang also participated in the research project, analyzing and interpreting the data. Weiming Li also participated in the design of this research and revised the manuscript. All authors participated in the discussion, reviewed and edited the manuscript, and approved the final manuscript.

Consent for publication: All authors approved the manuscript for publication.

Competing interests: The authors have declared that no competing interest exists. 
Acknowledgements: I would like to express my gratitude to everyone who helped me during the writing of the paper. Thank you very much for the comments provided by Tang and Li in this project, and they collaborated to complete the paper. I am grateful to my relatives, especially my parents. Although the project was not funded, they have always encouraged me to conduct academic research and supported me without complaint.

Authors' information: Yan Xu: Department of Clinical Laboratory, Hunan Aerospace Hospital, Changsha 410205, Hunan, China. E-mail 1272752827@qq.com. Ruiti Tang: Department of Clinical Laboratory, Hunan Aerospace Hospital, Changsha 410205, Hunan, China. E-mail 568299082@qq.com. Weiming Li: Department of orthopedics, Huazhong University of Science and Technology Union Shenzhen Hospital, Shenzhen 518000, Guangdong, China. E-mail liweiming@csu.edu.cn.

Funding: Not applicable.

Ethical Approval and Consent to participate: Not applicable

\section{References}

1. Feinstone SM, Kapikian AZ, Purceli RH, Hepatitis A. detection by immune electron microscopy of a viruslike antigen associated with acute illness. Science. 1973;182:1026-8. doi:10.1126/science.182.4116.1026.

2. Stuart DI, Ren J, Wang X, Rao Z, Fry EE. Hepatitis A Virus Capsid Structure. Cold Spring Harb Perspect Med 2019, 9, doi:10.1101/cshperspect.a031807.

3. Randazzo W, Sanchez G. Hepatitis A infections from food. J Appl Microbiol. 2020;129:1120-32. doi:10.1111/jam.14727.

4. Lima LR, De Almeida AJ, Tourinho Rdos S, Hasselmann B, Ximenez LL, De Paula VS. Evidence of hepatitis A virus person-to-person transmission in household outbreaks. PLoS One. 2014;9:e102925. doi:10.1371/journal.pone.0102925.

5. Nelson NP, Murphy TV, Hepatitis A. The Changing Epidemiology of Hepatitis A. Clin Liver Dis (Hoboken). 2013;2:227-30. doi:10.1002/cld.230.

6. Aggarwal R, Goel A, Hepatitis. A: epidemiology in resource-poor countries. Curr Opin Infect Dis. 2015;28:488-96. doi:10.1097/QC0.0000000000000188.

7. Jacobsen KH, Wiersma ST, Hepatitis A virus seroprevalence by age and world region, 1990 and 2005. Vaccine 2010, 28, 6653-6657, doi:10.1016/j.vaccine.2010.08.037.

8. Iorio N, John S, Hepatitis A. In StatPearls, Treasure Island (FL), 2021.

9. Xu ZY, Wang XY, Liu CQ, Li YT, Zhuang FC. Decline in the risk of hepatitis A virus infection in China, a country with booming economy and changing lifestyles. J Viral Hepat. 2008;15(Suppl 2):33-7. doi:10.1111/j.1365-2893.2008.01026.x.

10. Zheng H, Lu Y, Wang F, Cui F. Epidemiological Analysis on Hepatitis A in China During $2004 \sim 2006$ CHINESE JOURNAL OF VACCINES AND IMMUNIZATION 2007, 13, 453-456, 
doi:CNKI:SUN:ZGJM.0.2010-05-021.

11. Heavey E. Hepatitis. A takes hold in the community. Nursing. 2020;50:24-8. doi:10.1097/01.NURSE.0000668424.98515.8b.

12. China Public Health Science Data Center. HAV infection data from 2004 to 2017, https://www.phsciencedata.cn/Share/ky_sjml.jsp?id=8defcfc2-b9a4-4225-b92c-ebb002321 cea. Availabe online: (accessed on 30 August 2021).

13. Havelaar AH, Kirk MD, Torgerson PR, Gibb HJ, Hald T, Lake RJ, Praet N, Bellinger DC, de Silva NR, Gargouri N, et al. World Health Organization Global Estimates and Regional Comparisons of the Burden of Foodborne Disease in 2010. PLoS Med. 2015;12:e1001923. doi:10.1371/journal.pmed.1001923.

14. Jacobsen KH. Globalization and the Changing Epidemiology of Hepatitis A Virus. Cold Spring Harb Perspect Med 2018, 8, doi:10.1101/cshperspect.a031716.

15. Hu X, Collier MG, Xu F. Hepatitis A Outbreaks in Developed Countries: Detection, Control, and Prevention. Foodborne Pathog Dis. 2020;17:166-71. doi:10.1089/fpd.2019.2648.

16. Abutaleb A, Kottilil S, Hepatitis A. Epidemiology, Natural History, Unusual Clinical Manifestations, and Prevention. Gastroenterol Clin North Am. 2020;49:191-9. doi:10.1016/j.gtc.2020.01.002.

17. Shin EC, Jeong SH, Natural History. Clinical Manifestations, and Pathogenesis of Hepatitis A. Cold Spring Harb Perspect Med 2018, 8, doi:10.1101/cshperspect.a031708.

18. Croci L, De Medici D, Scalfaro C, Fiore A, Toti L. The survival of hepatitis A virus in fresh produce. Int J Food Microbiol. 2002;73:29-34. doi:10.1016/s0168-1605(01)00689-4.

19. Hettmann A, Juhasz G, Dencs A, Treso B, Rusvai E, Barabas E, Takacs M. Phylogenetic analysis of a transfusion-transmitted hepatitis A outbreak. Virus Genes. 2017;53:15-20. doi:10.1007/s11262-0161392-0.

20. Freidl GS, Sonder GJ, Bovee LP, Friesema IH, van Rijckevorsel GG, Ruijs WL, van Schie F, Siedenburg EC, Yang JY, Vennema H. Hepatitis A outbreak among men who have sex with men (MSM) predominantly linked with the EuroPride, the Netherlands, July 2016 to February 2017. Euro Surveill 2017, 22, doi:10.2807/1560-7917.ES.2017.22.8.30468.

21. Marrs T, Bruce KD, Logan K, Rivett DW, Perkin MR, Lack G, Flohr C. Is there an association between microbial exposure and food allergy? A systematic review. Pediatr Allergy Immunol. 2013;24(e318):311-20. doi:10.1111/pai.12064.

22. Cook N, Bertrand I, Gantzer C, Pinto RM, Bosch A. Persistence of Hepatitis A Virus in Fresh Produce and Production Environments, and the Effect of Disinfection Procedures: A Review. Food Environ Virol. 2018;10:253-62. doi:10.1007/s12560-018-9349-1.

23. Matheny SC, Kingery JE, Hepatitis A. Am Fam Physician 2012, 86, 1027-1034; quiz 1010-1022.

24. Young MK. The indications and safety of polyvalent immunoglobulin for post-exposure prophylaxis of hepatitis A, rubella and measles. Hum Vaccin Immunother. 2019;15:2060-5.

doi:10.1080/21645515.2019.1621148. 
25. Lin KY, Chen GJ, Lee YL, Huang YC, Cheng A, Sun HY, Chang SY, Liu CE, Hung CC. Hepatitis A virus infection and hepatitis $A$ vaccination in human immunodeficiency virus-positive patients: A review. World J Gastroenterol. 2017;23:3589-606. doi:10.3748/wjg.v23.i20.3589.

26. Shouval D, Immunization against Hepatitis A. Cold Spring Harb Perspect Med 2019, 9 , doi:10.1101/cshperspect.a031682.

27. Advisory Committee on Immunization. Fiore P, Wasley AE, Bell A. B.P. Prevention of hepatitis A through active or passive immunization: recommendations of the Advisory Committee on Immunization Practices (ACIP). MMWR Recomm Rep 2006, 55, 1-23.

28. Halliday ML, Kang LY, Zhou TK, Hu MD, Pan QC, Fu TY, Huang YS, Hu SL. An epidemic of hepatitis A attributable to the ingestion of raw clams in Shanghai, China. J Infect Dis. 1991;164:852-9. doi:10.1093/infdis/164.5.852.

\section{Figures}

Figure 1.

A

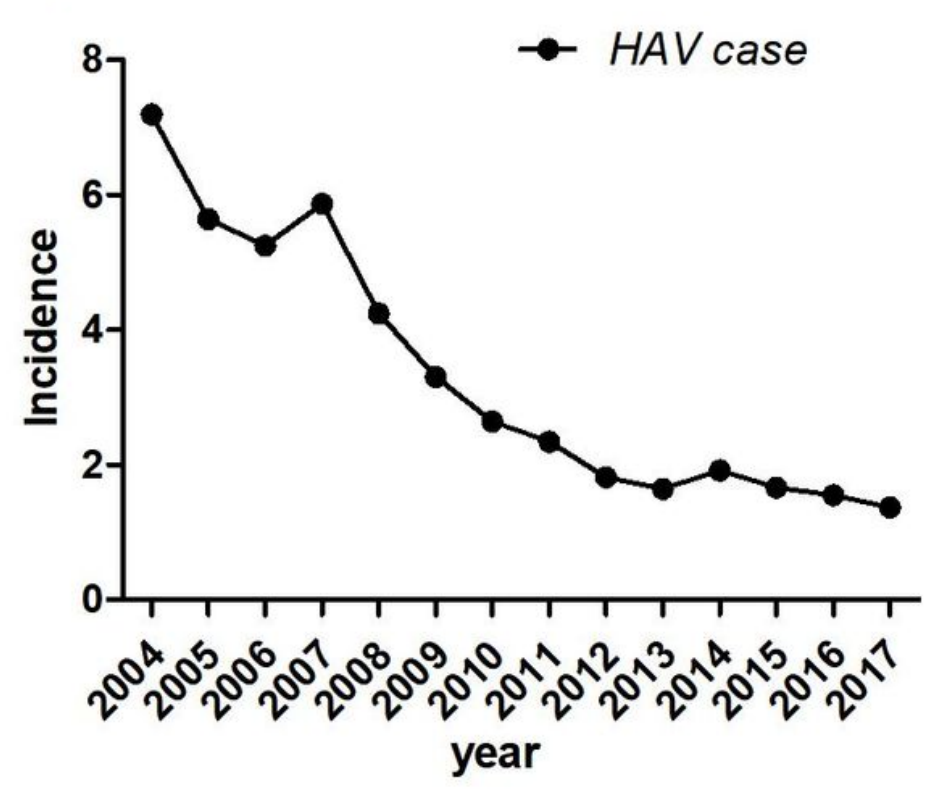

B

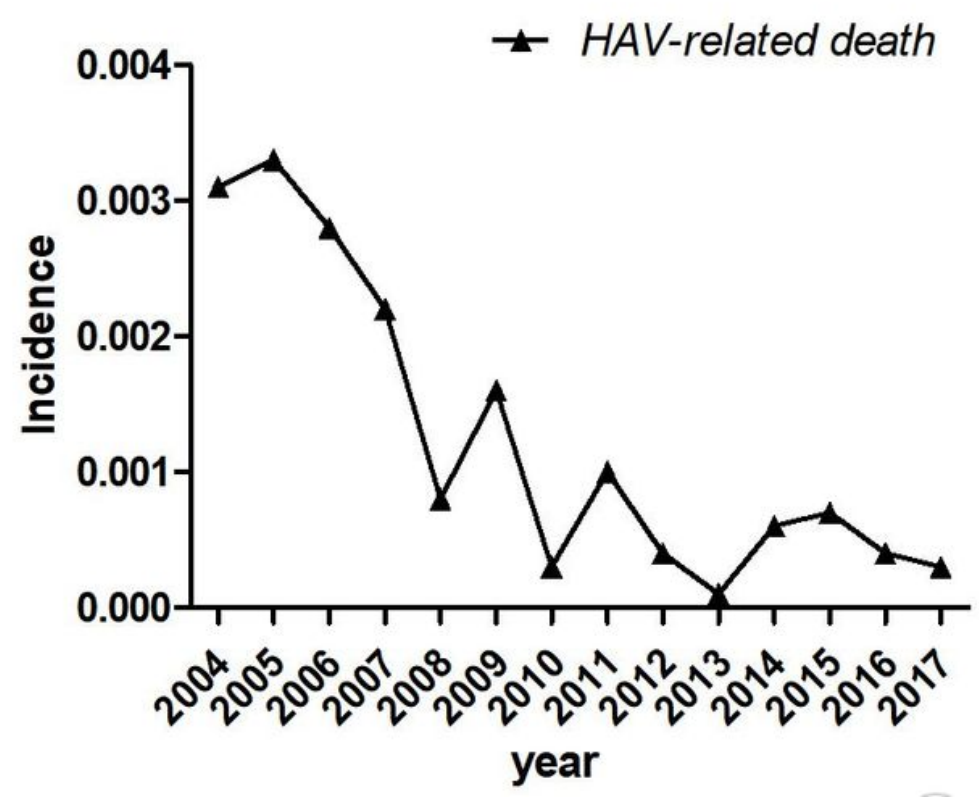

Figure 1

(A) The incidence of HAV cases $(1 / 100,000)$ in Chinese populations, 2004-2017. (B) The incidence of HAV-related death $(1 / 100,000)$ in Chinese populations, 2004-2017. 
A

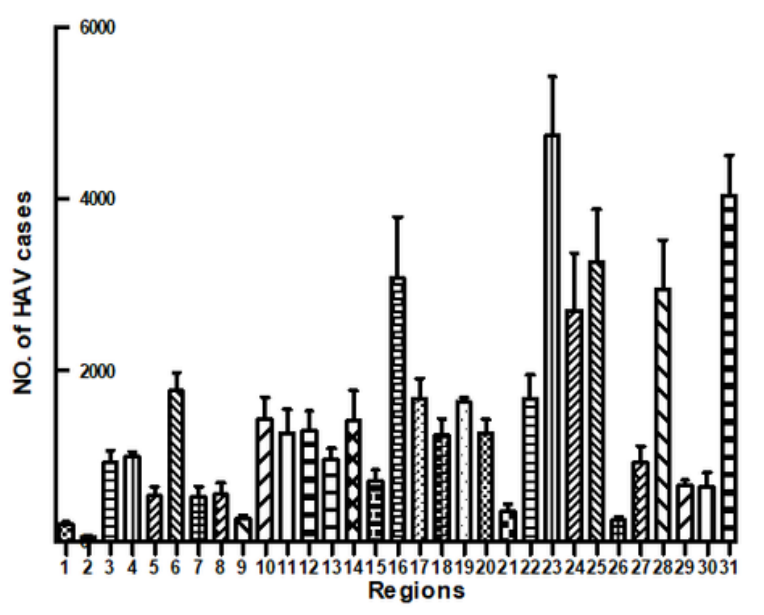

B

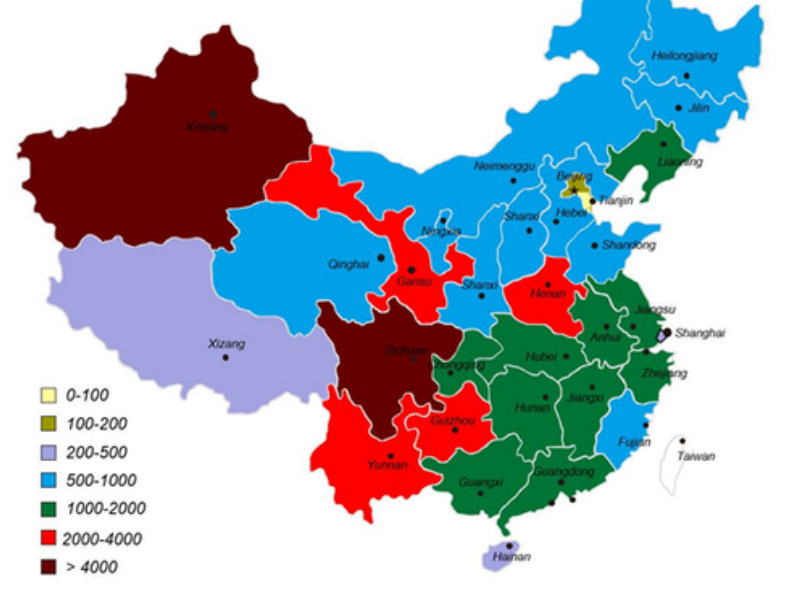

C

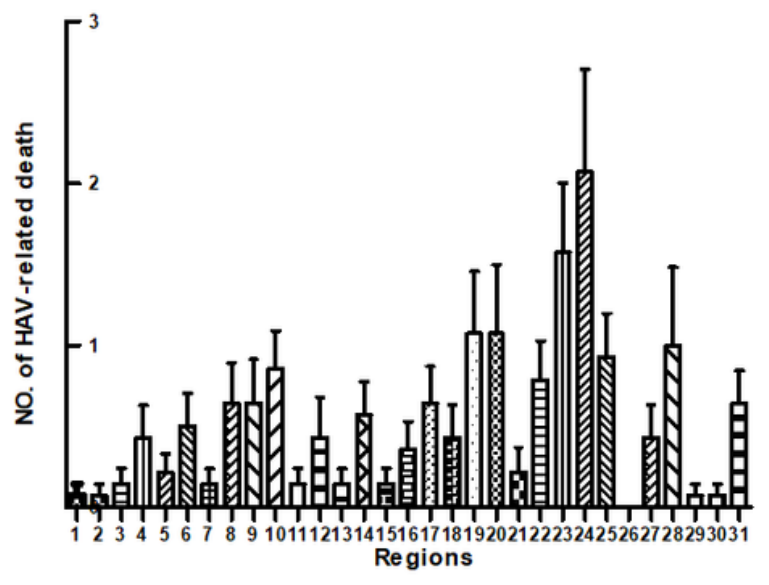

D

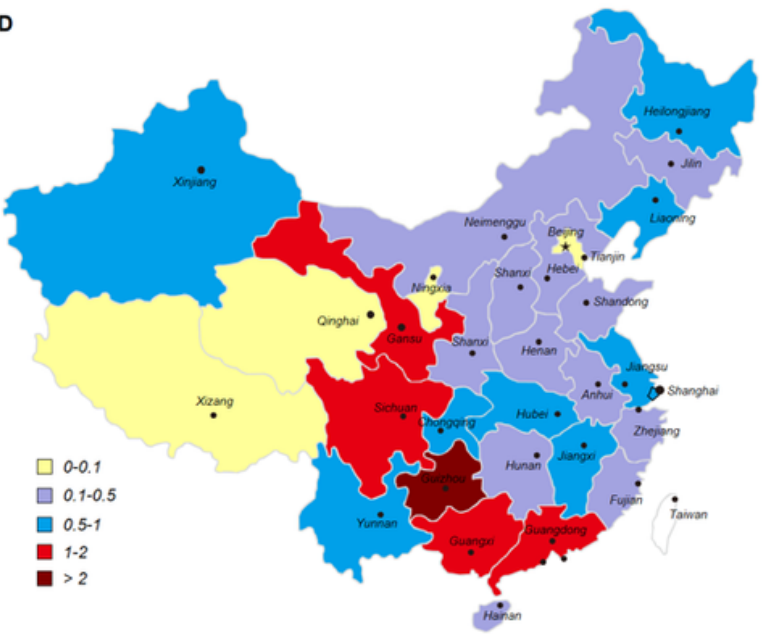

Figure 2

The average number (year) by regional distribution of HAV cases and HAV-related death in Chinese populations, 2004-2017 (A) (B) The average number (year) by regional distribution of HAV cases; (C) (D) The average number (year) by regional distribution of HAV-related death. The coordinate numbers on the $X$ axis of the Histogram (A) (C) represent different regions: 1-Beijing, 2-Tianjin, 3-Hebei, 4-Shanxi, 5-Inner Mongolia, 6-Liaoning, 7-Jilin, 8-Heilongjiang, 9-Shanghai, 10-Jiangsu, 11-Zhejiang, 12-Anhui, 13-Fujian, 14-Jiangxi, 15-Shandong, 16-Henan, 17-Hubei, 18-Hunan, 19-Guangdong, 20-Guangxi, 21-Hainan, 22Chongqing, 23-Sichuan, 24-Guizhou, 25-Yunnan, 26-Tibet, 27-Shaanxi, 28-Gansu, 29-Qinghai, 30-Ningxia, 31-Xinjiang. 
Figure 3

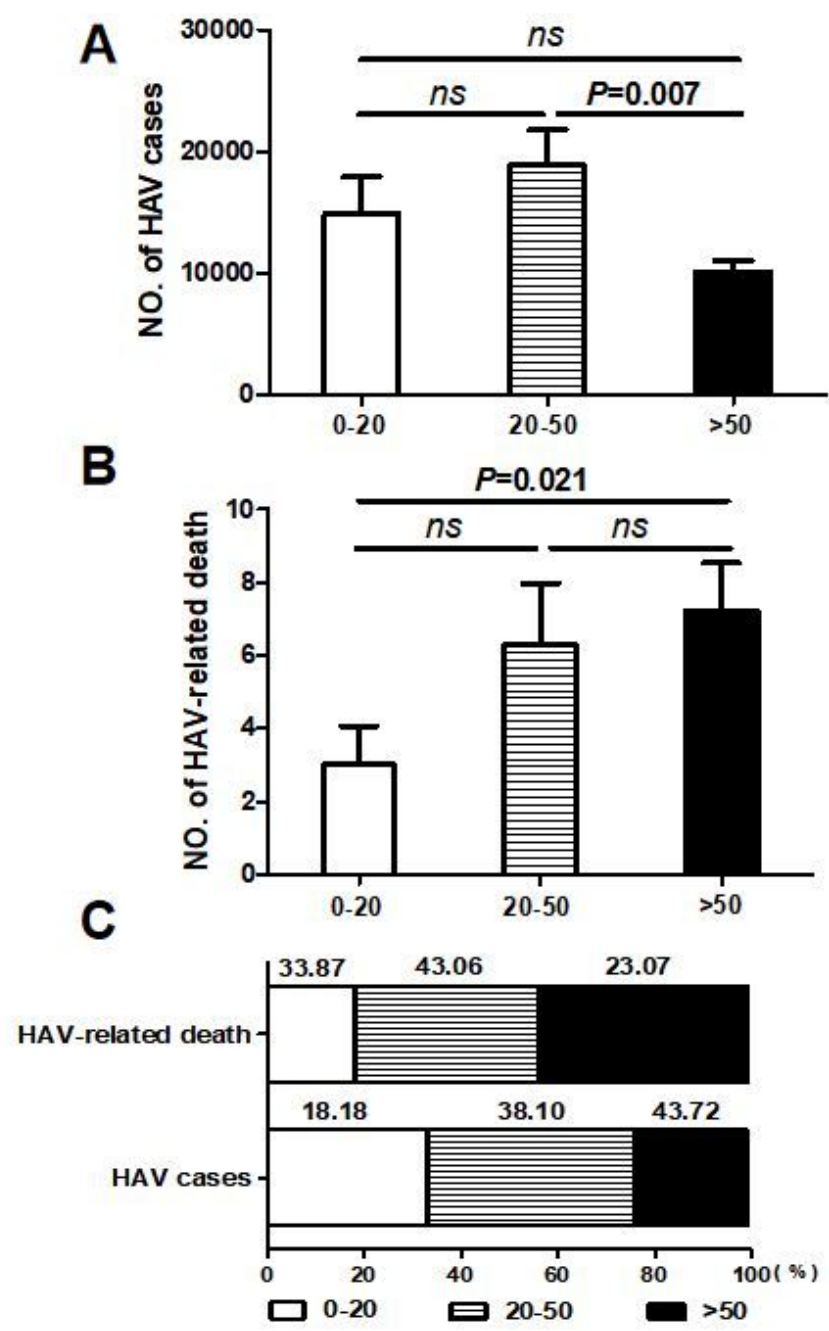

Figure 3

The averaged number (year) by age difference of HAV cases and HAV-related death in Chinese populations, 2004-2017 (A) HAV cases in Chinese populations, 2004-2017; (B) HAV-related death in Chinese populations, 2004-2017; (C) The percent distribution of HAV cases and HAV-related death.

\section{Figure 4}

A

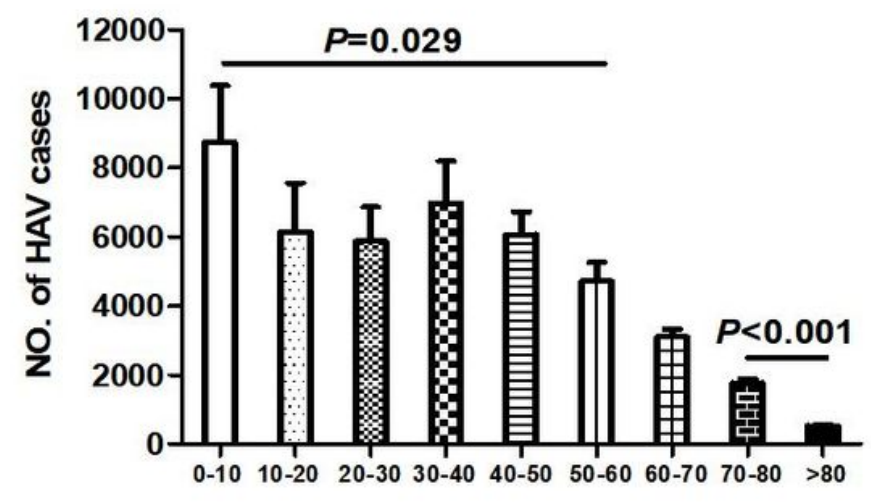

B

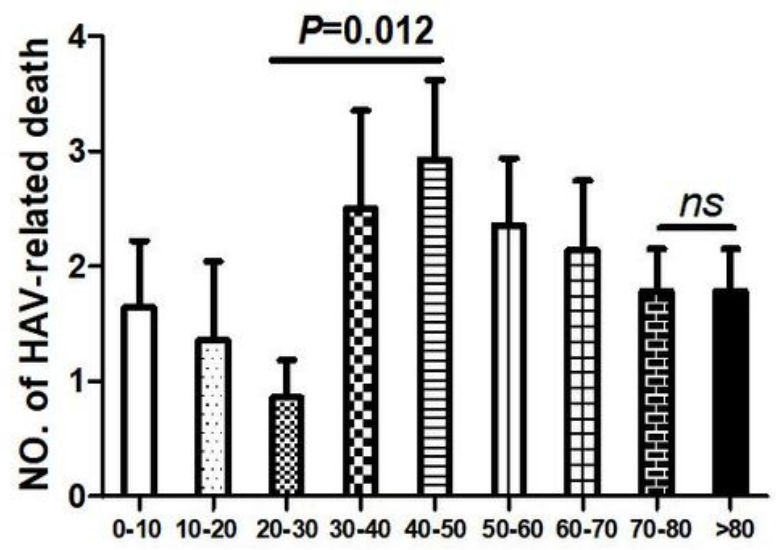




\section{Figure 4}

The averaged number (year) by age difference of HAV cases and HAV-related death in Chinese populations, 2004-2017 (A) HAV cases in Chinese populations, 2004-2017; (B) HAV-related death in Chinese populations, 2004-2017.

\section{Supplementary Files}

This is a list of supplementary files associated with this preprint. Click to download.

- geographicalmap.pptx 